\title{
Design and manufacturing status of Trim Coils for the Wendelstein 7-X stellarator experiment
}

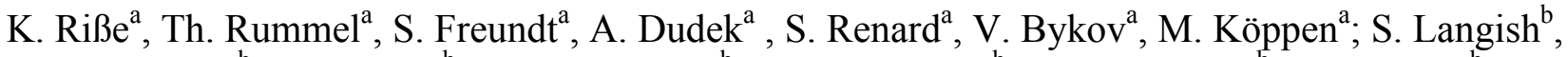 \\ G.H. Neilson ${ }^{\mathrm{b}}$, Th. Brown ${ }^{\mathrm{b}}$, J. Chrzanowski $^{\mathrm{b}}$, M. Mardenfeld ${ }^{\mathrm{b}}$, F. Malinowski ${ }^{\mathrm{b}}$, A. Khodak ${ }^{\mathrm{b}}, \mathrm{X}$. \\ Zhao $^{\text {b }}$, G.Eksaa ${ }^{\mathrm{c}}$ \\ ${ }^{a}$ Max-Planck-Institut für Plasmaphysik, Greifswald Germany \\ ${ }^{b}$ Princeton Plasma Physics Laboratory, Princeton NJ USA \\ ${ }^{c}$ Everson Tesla Inc., Nazareth PA USA
}

\begin{abstract}
The stellarator fusion experiment Wendelstein 7-X (W7-X) is currently under construction at the Max-PlanckInstitut für Plasmaphysik in Greifswald, Germany. The main magnetic field will be provided by a superconducting magnet system which generates a fivefold toroidal periodic magnetic field. However, unavoidable tolerances can result in small deviations of the magnetic field which disturb the toroidal periodicity. In order to have a tool to influence these field errors five additional normal conducting trim coils were designed to allow fine tuning of the main magnetic field during plasma operation. In the frame of an international cooperation the trim coils will be contributed by the US partners. Princeton Plasma Physics Laboratory has accomplished several tasks to develop the final design ready for manufacturing e.g. detailed manufacturing design for the winding and for the coil connection area. The design work was accompanied by a detailed analysis of resulting forces and moments to prove the design. The manufacturing of the coils is running at Everson Tesla Inc; the first two coils were received at IPP.
\end{abstract}

Keywords: magnet, magnetic field, trim coil, stellarator

\section{Introduction}

The stellarator fusion experiment Wendelstein 7-X (W7-X) is currently under construction at the Max-PlanckInstitut für Plasmaphysik (IPP) in Greifswald, Germany. [1,2] The main magnetic field will be provided by a superconducting magnet system consisting of 70 coils distributed in five identical modules. The coil system generates a fivefold toroidal periodic magnetic field. Unavoidable tolerances can result in small deviations of the magnetic field which disturb the toroidal periodicity. In order to have a tool to influence these field errors five additional normal conducting trim coils were designed to allow fine tuning of the main magnetic field during plasma operation. Due to construction space restrictions two different coil types were developed, four type A coils and one coil of type B. The coils will be mounted on the outer cryostat wall, one coil per each of the five W7-X modules (see Fig.1). The supports were designed and procured in the industry by IPP.

In the frame of an international cooperation which started in November 2010, the US partners Princeton Plasma Physics Laboratory (PPPL), Oak Ridge Laboratories (ORNL) and the Los Alamos National Laboratories (LANL) received a 3 year funding program to support the stellarator research at IPP. The US partners will contribute the five trim coils and their power supplies within the frame of this support program.

\section{Purpose of trim coils}

\subsection{Error field compensation}

The stellarator field is very sensitive to non-systematic deviations which will create asymmetric magnetic field errors. The source of such asymmetric field errors can be: deviations from ideal coil shapes due to manufacturing tolerances, small asymmetric misalignments of coils or modules during assembly, non-systematic displacements and deformations under magnetic loads due to different stiffness of coil casings or support structure or ferromagnetic materials near to the plasma.

Such individual deviations break the symmetry and cause error field components which are already resonant in the lowest order to $l=1$. Resonant means that the Fourier components of the error field are in phase with the field line. Such field perturbations lead to additional magnetic islands and asymmetric thermal loads on the divertor targets. Additionally, untoward effects can result as reduction of plasma radius with creation of additional island with different periodicity and generation of stochastic regions or shift of the plasma centre. The requirement of good plasma confinement and reliable divertor operation limits the relative amplitude of error field components to be below B/B00 $<2 \times 10-4$, where B00 is the average magnetic field strength on the magnetic axis. [3] 


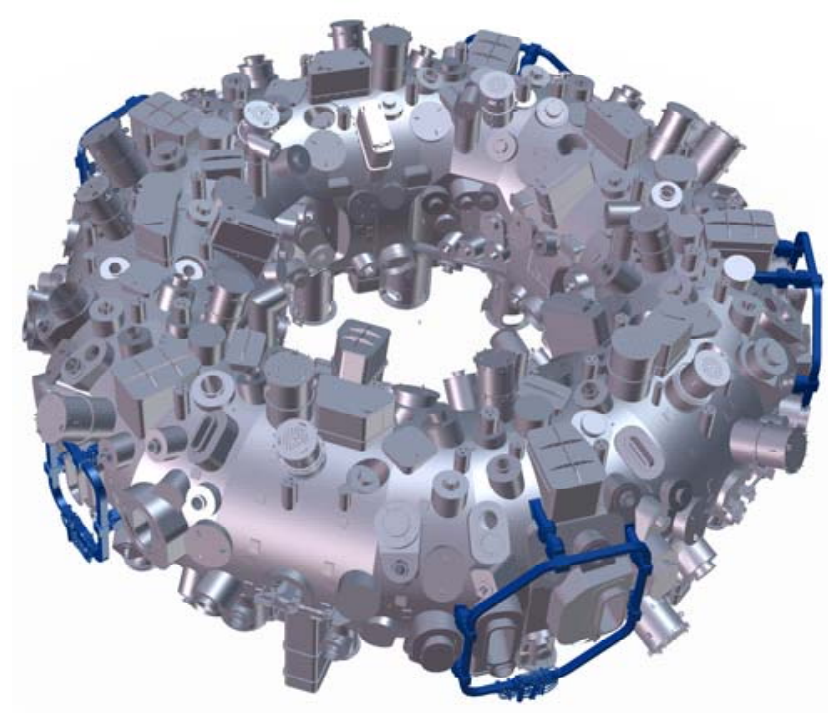

Fig.1 W7-X with trim coils - Type A coil at foreground

\subsection{Purpose of trim coils}

Despite all efforts to correct the magnetic field errors during assembly some uncertainties remain. The trim coil system provides a tool to compensate remaining field errors by adding Fourier components with the same amplitude but opposite phase angle. In addition, such trim coils allow a higher experimental flexibility by influencing the magnetic field, e.g. creating magnetic islands or influencing the magnetic axis. [3]

\section{Design of trim coils}

The coil design is based on requirements for the necessary magnetic flux density to be created on the plasma axis in the middle of the modules and the available construction space on the outer vessel. Different shapes and positions for the trim coils were investigated, and at the end it had been decided to install normal conducting planar coils at the positions with bean-shaped plasma cross-section. The recent design is a compromise between available construction space close to the outer vessel, power consumption and manufacturing costs. The type B coil is different due to space restrictions created by the large Neutral Beam Injection boxes. Five support beams for type A (four for type B), welded to the outer vessel and clamped via silicon rubber shims to the coil, will keep the coil in position.

\subsection{Electrical parameters}

The electrical parameters of the trim coils are presented in Table 1. The type A coil has a nearly rectangular shape with dimensions of $3.5 \mathrm{~m} \times 3.3 \mathrm{~m}$. The $110 \times 151 \mathrm{~mm}^{2}$ coil cross section is comparably lean. The type B coil, with dimensions of $2.2 \mathrm{~m} \times 2.8 \mathrm{~m}$, is smaller. To compensate for the smaller size the B coil has with 72 turns, a larger number of turns and an increased operational current. The coils are made of an oxygen free hollow copper extrusion, with a square cross section, side lengths of $16.26 \mathrm{~mm}$ and an internal coolant hole diameter of $10.2 \mathrm{~mm}$.

Table 1: Electrical parameters

\begin{tabular}{lrrr}
\hline & Coil Type & A & B \\
\hline Number of turns in 8 & & 48 & 72 \\
pancakes & $\mathrm{mH}$ & 17.8 & 25.1 \\
Inductance & $\mathrm{kA}$ & 1.8 & 1.95 \\
Nominal current & $\mathrm{kA}$ & 86 & 140 \\
Total coil current & $\mathrm{m}$ & 556 & 617 \\
Coil conductor length & $\mathrm{m} \Omega$ & 51 & 56 \\
Electrical resistance & $\mathrm{V}$ & 140 & 173 \\
Nominal voltage & $\mathrm{kW}$ & 200 & 260 \\
Dissipated power & & & \\
\hline
\end{tabular}

The insulation system of the coils is designed to withstand the nominal voltages and the test voltages. The test voltage against ground is $2 \mathrm{kV}$ DC and requires an insulation resistance of $1 \mathrm{G} \mathrm{Ohm}$. The tests shall be done with empty water cooling lines. To test the interturn insulation a test voltage of $800 \mathrm{~V}$ shall be applied across the coil terminals.

\subsection{Hydraulic parameters}


The coil cooling is designed to have a cooling capacity of $200 \mathrm{~kW}$ for the type A coil and $260 \mathrm{~kW}$ for type B by keeping a temperature difference between inlet and outlet of $30 \mathrm{~K}$. Table 2 explains the main hydraulic parameters. The maximum operational pressure of type A coils is 17 bars and 21 bars for type B respectively. A $20 \%$ safety margin is included to cover some uncertainties. The static pressure in the cooling circuit, the hydraulic resistances of the cooling water return lines, and a safety valve resistance were considered in these values.

Table 2: Hydraulic parameters

\begin{tabular}{lccc}
\hline & Coil Type & A & B \\
\hline Temperature inlet max. & ${ }^{\circ} \mathrm{C}$ & \multicolumn{2}{c}{30} \\
Delta Temperature & ${ }^{\circ} \mathrm{C}$ & \multicolumn{2}{c}{30} \\
Temperature outlet max. & ${ }^{\circ} \mathrm{C}$ & \multicolumn{2}{c}{70} \\
Pressure drop nominal & bar & 6 & 10 \\
Max. operational pressure & bar & 17 & 21 \\
Test pressure & bar & 25 & 31 \\
\hline
\end{tabular}

Deionised water will be used for the coil cooling, which provides a very low electrical conductivity of $<5 \mu \mathrm{S} / \mathrm{cm}$.

\subsection{Instrumentation}

Platinum temperature sensors of type PT100 per DIN 60751 will be installed to enable control of the cooling water temperature.

The temperature of the cooling water inlet will be monitored by placing one temperature sensor on the inlet manifold. The instrumentation of the coil outlets must ensure that any atypical conductor temperature can be detected. For this reason, one temperature sensor each will be positioned onto the conductor profile at the four outlets. These temperature sensors will be placed on the pancake crossovers, which carry the outlet water of two pancakes.

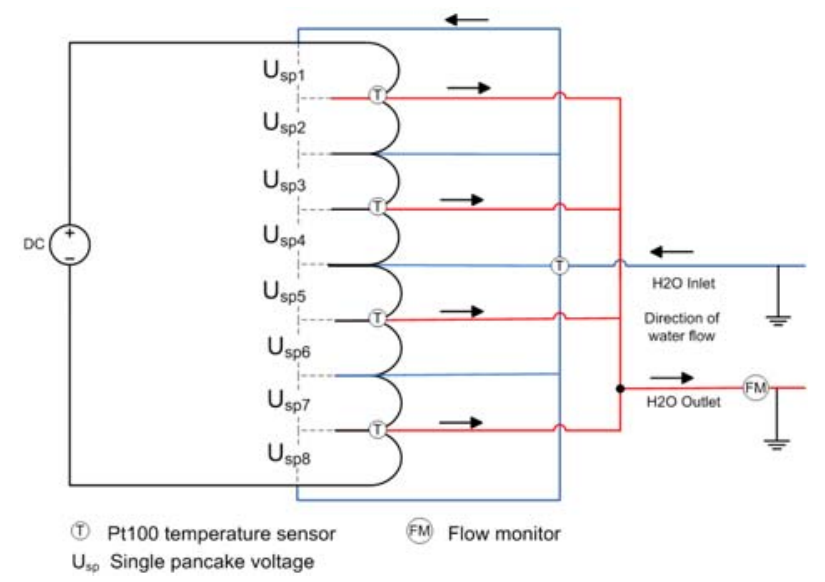

Fig.2 Schematic cooling circuit with instrumentation.

To control the pancake temperatures a voltage tap system will be installed as a safety system to assure proper cooling in each pancake. The voltage taps allow voltage readings of each pancake during coil operation. Knowing the current in the coil and the voltage the related pancake temperature can be evaluated in the central control system. Trip off points will be set for allowed temperatures in the pancake as well as for the temperature differences between the pancakes. To limit the stress in the coil the fault scenario is set to $\geq 2.5^{\circ} \mathrm{C}$ difference in the mean temperature between pancakes.

In the hydraulic outlet line of each coil a flow monitor will control if the required cooling water flow is present. The flow monitor signal prerequisites electric current operation.

\subsection{Coil position and tolerances}

Each trim coil has a dedicated CAD position which is free from collisions. A collision control was necessary due to the large number of neighboring components already close to the coil position. The collision control is based on the following considered tolerances:

- Trim coil manufacturing tolerance $\pm 5 \mathrm{~mm}$

- Trim coil movements during operation $\pm 5 \mathrm{~mm}$

- Trim coil assembly tolerance of $\pm 3 \mathrm{~mm}$

- Outer vessel manufacturing and assembly tolerance $\pm 22 \mathrm{~mm}$ ( adjusted by shims) 
- Deflections of outer vessel $<18 \mathrm{~mm}$ during baking phase of the plasma vessel, cooling down or plasma operation

- Safety margin of $5 \mathrm{~mm}$ plus remaining distances to neighboring components of $5 \mathrm{~mm}$

Due to the distance of app. $1.5 \mathrm{~m}$ between plasma and trim coils, the manufacturing and assembly tolerances of few millimeters have a minor influence on the magnetic field correction.

\subsection{Coil supports}

Five coil supports will fix the type A coil (four for type B) to the outer cryostat wall. The support consist of one beam which is welded to the outer cryostat wall, one shim element allows both length and angular adjustments, and the clamp (see Fig. 3).

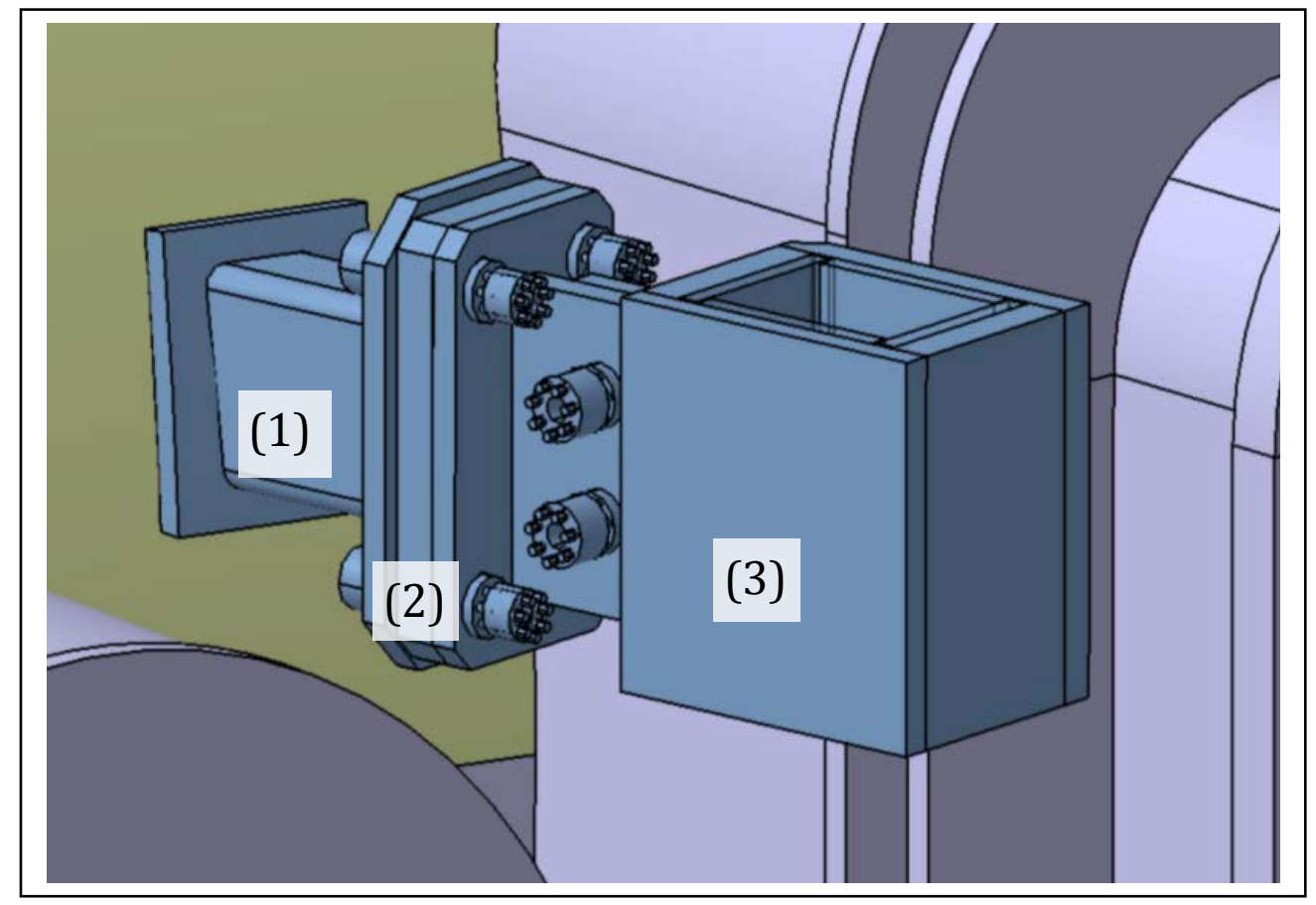

Fig.3 Schematic view of one coil support, shown without coil, (1) beam, (2) adjustable shim plate, (3) clamp with elastomeric shims.

The clamp will be equipped with elastomeric pads which will transfer the clamping forces to the coil. In the main clamping direction a pressure of about $1 \mathrm{MPa}$ will be adjusted by implementing movable stamps, while in the second direction 0.5 MPa will be applied [4]. Nuts with multi-jackbolt tensioners will be used to fix the clamp on the outer vessel. These nuts allow applying a higher torque with smaller hand tools.

\section{Structural Analysis}

Key mechanical criteria for acceptable operation of the trim coils are limitations on overall deflection as well as shearing stresses and (secondary) normal strains across the conductor insulation bond. The prior is restricted due to space considerations, and the latter to prevent insulation delamination after many operational cycles. Other structural considerations, such as hoop stresses in the conductor and in-ply stresses of the laminate tend to easily meet the requirements.

The maximum operational deflection is on the order of $4.5 \mathrm{~mm}$, compared to an allowable of $5 \mathrm{~mm}$ (see Fig. 4). The maximum shearing stress across bond planes is of order $16 \mathrm{MPa}$, compared to a tested life of $24 \mathrm{MPa}$ at 60,000 cycles for the cyanate ester/ epoxy blend impregnated insulation [5]. These results consider the effects of Lorentz forces from steady state self fields and stellarator coil fields, gravity loads, deformation imposed by outer vessel and thermal effects. 


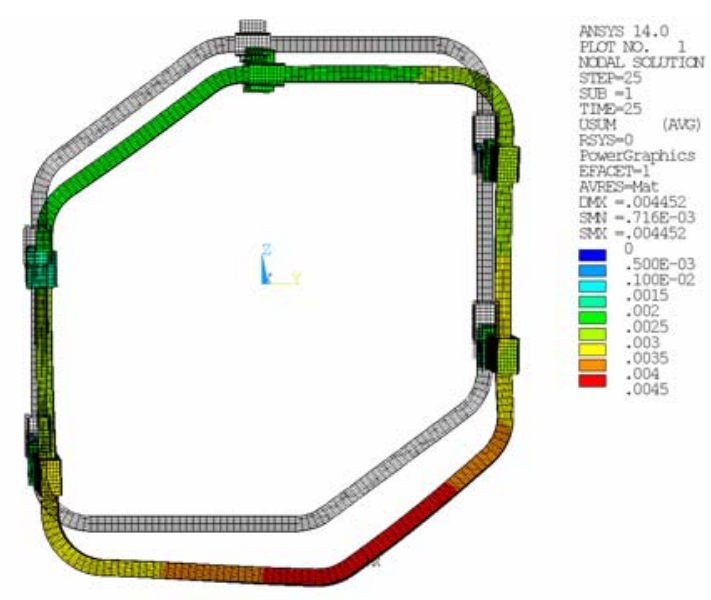

Fig.4 Visual amplified (factor 100) displacements of type A coil during operation

\section{1 Electromagnetic Effects}

The electromagnetic forces on the coil create a behavior which is quite similar to a distributed load on a beam. Consequently, electromagnetically induced stresses are relatively benign, and internally reacted in directions in which the coil is strong (see Fig. 5). However, due to the distance between support legs, this net "beam bending" is responsible for a greater proportion of the deflection.

Note that the stellarator field coils and noncircular geometry of the coil result in nonuniform loads, and bending as well as hoop tension.

The largest net magnetic force occurs during the "High Iota" configuration of W7-X. Trim coil B will be subjected to a total force of $24.6 \mathrm{kN}$ with local force densities of approx. $74 \mathrm{MN} / \mathrm{m}^{3}$. Approx. $17 \mathrm{kN}$ will act as a total force on trim coil $\mathrm{A}$ and the local force density maximum reaches about $35 \mathrm{MN} / \mathrm{m}^{3}$.

Due to the position of the trim coils with respect to stellarator symmetry, the inductive coupling to the W7-X superconducting coils is small (below $0.1 \%$ ). Therefore, even quick changes of the currents, e.g. during a fast discharge of the super conducting coils, will have only a small influence on the trim coil currents.
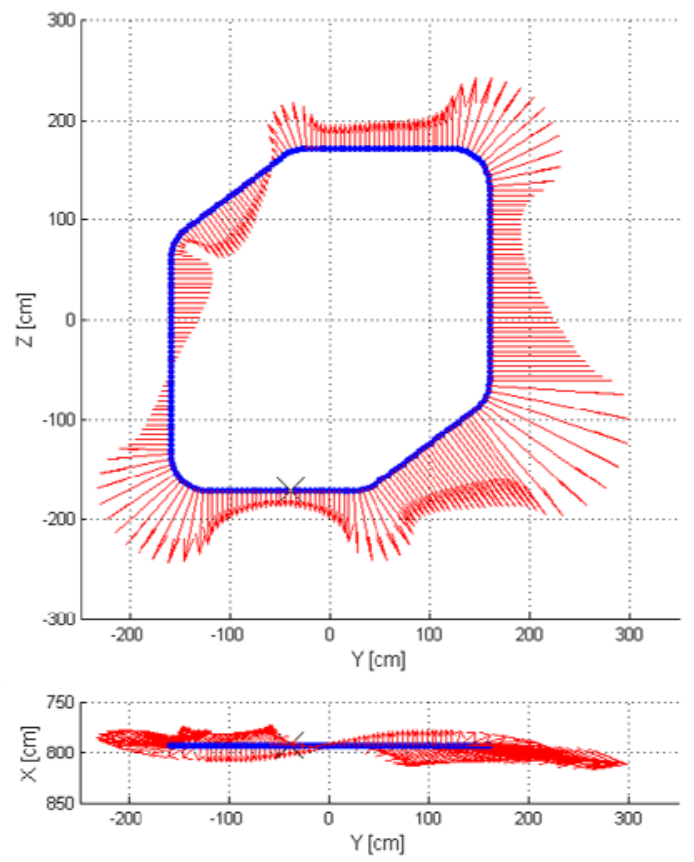

Fig.5 Electromagnetic forces on trim coil A for "High Iota" configuration of W7-X

\section{2 Thermal Effects}

The $30 \mathrm{~K}$ temperature rise across the inlet and outlet of the coil occurs across the width of the cross section near the lead region. Furthermore, the continuous helical winding of the conductors creates locations where adjacent turns are $5^{\circ} \mathrm{C}$ apart, separately only by thin insulation layers $(\sim 1 \mathrm{~mm})$. These effects distort the cross section of the coil from a rectangle to a trapezoid, and conductors on the inner diameter of the coil tend to expand more than the outer diameter. 
Collectively this induces bending stresses and tension normal to the copper insulation boundary. These effects ultimately limit the life of the coil to 60,000 cycles.

\section{3 Coil Supports}

The length of the support beams and the $15 \mathrm{~mm}$ thick elastomeric shims inside of the support clamps provide the required flexibility for strain relief. Simulation during the High Iota Configuration with support beams fixed at the outer cryostat wall estimate reaction forces of $10 \mathrm{kN}$ and torsion torque of $62 \mathrm{Nm}$ in X-direction and bending torques of 3600 $\mathrm{Nm}$ in Y-direction.

\section{Project status}

After the start of the trim coil project in November 2010, the US partners developed the manufacturing design and performed a structural analysis to prove the coil design. Mid of 2011 the call for tender was send out, and the contract for the coil manufacturing was awarded to the company Everson-Tesla Inc. in Nazareth PA, USA in September 2011. The winding of the first coil started after procurement of tools, conductor and materials in February 2012. Currently two type A coils are at IPP. The assembly of the clamps is ongoing for the first coil, to be ready for assembly onto the outer vessel in October 2012. The type B coil is the last coil to be delivered, expected in February 2013. The general W7-X assembly schedule requires the coil assembly in specific time slots, e.g. to install the coils before the cooling pipe assembly starts on the outer vessel or before the large Electron Cyclotron Resonance Heating towers will be placed in the torus hall. The procurement of the coil supports, clamps, bolts and nuts will be finished by end of October 2012 .

\section{Summary}

The W7-X stellarator will be equipped with five additional trim coils to be mounted on the outer cryostat wall. The trim coils will allow compensation for remaining field errors and enable a higher experimental flexibility by influencing the magnetic field. The trim coils are normal conducting water cooled copper coils and individually controlled by a power supply. The trim coils and the related power supplies will be contributed within the frame of a collaboration program between the PPPL, Oak Ridge National Laboratory and the Wendelstein 7-X project and are funded by the U.S. Department of Energy. The company Everson Tesla Inc. has been manufacturing the five trim coils. The first two type A coils are already at IPP. The first coils shall be finally assembled to the W7-X outer vessel in October 2012.

\section{Acknowledgments}

The collaboration for the trim coil project with the US associations Princeton Plasma Physics Laboratory, Oak Ridge Laboratories and the vendor Everson Tesla Inc. as well as the funding by the US Department of Energy is gratefully acknowledged. Sincere thanks to my colleagues from the W7-X team for their support and contributions to this paper.

[1] L. Wegener, "Status of Wendelstein 7-X Construction", Fusion Engineering and Design, 84 (2009), pp. 106-112.

[2] H.-S. Bosch "Construction of Wendelstein 7-X - Engineering a Steady-State Stellarator", IEEE Trans. Plasma Sceince 38 (2010) $265-273$.

[3] J. Kißlinger and T. Andreeva, "Correction possibilities of magnetic field errors in Wendelstein 7-X," in Fusion Engineering and Design 74 (2005) 623-626.

[4] S. Freundt, "FE analyses and tests in support of Wendelstein 7 X trim coil development", Fusion Engineering and Design, Proceedings of 27 SOFT

[5] Naseem A. Munshi, "Radiation Resistant Electrical Insulation Qualified for ITER TF Coils",IEEE Trans. on applied superconductivity, Vol. 23, No. 3, 2013 